\title{
Psiquiatria Biológica e Psicofarmacologia: a formação de uma rede tecnocientífica
}

I ${ }^{1}$ Marcela Peralva Aguiar, ${ }^{2}$ Francisco Javier Guerrero Ortega |

Resumo: A Psiquiatria Biológica emerge nos anos de 1980, nos Estados Unidos, com a publicação do DSM-III (Diagnostic and Statistical Manual of Mental Disorders). Este manual tem a proposta de ser a-teórico e apresentar descriçóes objetivas dos, então, chamados "distúrbios mentais" a partir de um critério classificatório que não inclui discussões acerca da etiologia destes "distúrbios". Isto implica que, apesar da denominação biológica conferida a esta vertente psiquiátrica, não houve nenhuma descoberta acerca da etiologia biológica destes distúrbios que justificasse sua denominação e seu estrondoso sucesso mundial. Neste artigo, pretendemos analisar as modificaçōes ocorridas na psiquiatria norte-americana a partir da introdução do DSM-III, dos primeiros medicamentos psicotrópicos e o subsequente papel desempenhado pela indústria farmacêutica neste processo. Desse modo, o que está em análise neste artigo é que, ao se apropriar dos medicamentos psicotrópicos como primeira opção terapêutica, a Psiquiatria Biológica cria uma aliança com a indústria farmacêutica que a insere numa rede tecnocientífica que dificulta saber os limites entre Psiquiatria Biológica, Psicofarmacologia e Indústria farmacêutica.
${ }^{1}$ Instituto de Medicina Social, Universidade do Estado do Rio de Janeiro. Rio de Janeiro-RJ, Brasil (marcelaperalva@gmail.com).

2 Instituto de Medicina Social, Universidade do Estado do Rio de Janeiro. Rio de Janeiro-RJ, Brasil (fjortega2@gmail.com). 
Ao longo de sua história, a psiquiatria constituiu seu arcabouço teóricometodológico a partir de diversas teorias que podemos condensar em duas vertentes principais: moral/humanista e fisicalista/biológica. Isto não significa que as teorias morais/humanistas e as fisicalistas/biológicas formem dois blocos homogêneos de atuação, mas sim que esta divisão nos permite reunir vertentes que possuem um arcabouço teórico-metodológico comum em um mesmo grupo e que em determinados momentos da história da psiquiatria um desses grupos obteve hegemonia no campo sem jamais eliminar o outro (BYNUM, W.F.; PORTER, R.; SHEPHERD, M., 1985; VALENSTEIN, 1998; SWAIN, 1994; SCULL, 1981, 2005, 2015; YOUNG, 1995; SHORTER, 1997; SERPA JR., 1998, 2000; BEZERRA JR., 2000; HEALY, 2002, 2003; RUSSO; VENANCIO, 2006; ROSENBERG, 2006).

Dentro desta perspectiva, é possível afirmar que a psiquiatria se constitui no século XVIII a partir de um referencial moral/humanista e que, ao final do século XIX, este referencial perderá espaço para as teorias fisicalistas/biológicas, que tentavam explicar a doença mental exclusivamente com base nestes fatores. A partir do segundo pós-guerra, estas teorias de cunho fisicalista perdem prestígio e novamente ganham destaque as de cunho moral-humanista, que percebiam a doença mental como uma doença multifatorial. Em fins do século XX, as teorias fisicalistas retomam a dominância do campo.

A chamada Psiquiatria Biológica, representante desta última retomada, ascende como vertente hegemônica, nos Estados Unidos, em 1980, a partir da publicação do DSM-III (Diagnostic and Statistical Manual of Mental Disorders). Este manual apresenta uma abordagem que se pretende a-teórica quanto à etiologia dos então chamados distúrbios mentais (ASSOCIAÇÃO AMERICANA DE PSIQUIATRIA, 1986; KIRK; KUTCHINS, 1992; KUTCHINS; KIRK, 2003; ROSENBERG, 2006; RUSSO;VENANCIO, 2006). Isto implica que, apesar da denominaçáo biológica conferida a esta vertente, a sua ascensão não esteve vinculada diretamente a nenhuma descoberta acerca da origem biológica dos distúrbios mentais como se poderia crer, pois até aquele momento nenhum marcador biológico havia sido encontrado para nenhum dos distúrbios mentais classificados em seu manual. E, vale dizer, que esta situação não mudou até o presente momento. 
No nível da investigação empírica, a hipótese de que a doença mental envolve anomalias cerebrais vem alimentando a busca de marcadores biológicos a fim de se distinguir a "normalidade" e a patologia. Dessa forma, seria a partir da localização das áreas cerebrais que apresentassem alguma forma de patologia que tratamentos específcos viriam a ser desenvolvidos. No entanto, até mesmo os defensores mais ferrenhos desta abordagem neurobiológica reconhecem que os biomarcadores permanecem "teimosamente fora de alcance" (HYMAN, 2008, p. 890). Em 2002, em uma contribuição para a preparação da quinta edição do DSM (lançado em 2013), um grupo de psiquiatras biológicos proeminentes observou que a psiquiatria não tinha até o momento "conseguido identificar um único marcador neurobiológico fenotípico ou gene que fosse útil para fazer um diagnóstico de um transtorno psiquiátrico ou para prever a resposta ao tratamento psicofarmacológico" (CHARNEY et al., 2002, p. 33). Mais de uma década depois, a situação demonstra não ter mudado (SERPA JR., 1998; CHARNEY et al., 2002; HYMAN, 2008; WALSH et al., 2011; MAYBERG, 2014; INSEL et al., 2010; INSEL, 2013; KAPUR; PHILLIPS; INSEL, 2012; KIRMAYER; CRAFA, 2014; ROSE, 2013b; GABRIELI; GHOSH; WHITFIELD-GABRIELI, 2015; ROSE; ABI-RACHED, 2013).

Assim, um artigo de 2011 sobre os desafios envolvidos na busca de biomarcadores do autismo concluiu que "apesar de grandes avanços na compreensão científica básica do autismo, comparativamente, pouco foi conseguido até a data no que diz respeito a traduzir a evidência resultante em biomarcadores clinicamente úteis" (WALSH et al., 2011, p. 609-610). E em 2014, a neuropsiquiatra da Universidade de Emory, Helen S. Mayberg, uma figura importante no campo da neuroimagem da depressão, admitiu com pessimismo que "as afirmações de alguns clínicos de que podem usar de forma confiável exames cerebrais estruturais ou funcionais" para objetivos de diagnóstico e tratamentos "não tem base médica ou científica". E acrescenta que tais alegaçôes se encontram "além do escopo da pesquisa atual e dão falsas esperanças para os pacientes e suas famílias” (MAYBERG, 2014, p. S34).

Um grupo de pesquisadores considerou que uma das razóes para essa falha residiria, em parte, nas categorias para as quais os biomarcadores estão sendo pesquisados, que são aquelas fornecidas pelo DSM e CID, ou seja, os manuais da Associação Psiquiátrica Americana (APA) e a Classificação Internacional de Doenças (CID) da OMS. Daí, a iniciativa do National Institute for Mental 
Health (NIMH) norte-americano, lançada em 2011, de afastar-se das categorias do DSM e desenvolver o Projeto Research Domain Criteria (RDOC), destinado a transformar o diagnóstico psiquiátrico através da convergência da genética, neuroimagem e ciências cognitivas (INSEL et al., 2010; INSEL, 2013; KAPUR; PHILLIPS; INSEL, 2012). O RDOC representa uma nova perspectiva na busca de marcadores neurobiológicos, mas não um novo ponto de partida radical.

$\mathrm{Na}$ verdade, o projeto mantém intacta a visão neurobiológica tradicional do distúrbio/transtorno mental, com seu foco em mecanismos biológicos discretos em detrimento de uma abordagem "ecossocial" mais integrada (KIRMAYER; CRAFA, 2014). Em RDOC, os marcadores biológicos deixarão de ser acoplados com categorias do DSM, mas as doenças mentais permanecerão definidas como "transtornos biológicos que envolvem os circuitos cerebrais que implicam domínios específicos da cognição, emoção ou comportamento" (INSEL, 2013). Para esses pesquisadores, o problema seria que a elucidação da etiologia desses transtornos demandaria "confiança no cérebro, e não no DSM" (ROSE, 2013, p. 10).

Contudo, a confiança no cérebro não tem produzido até agora praticamente nenhum resultado de utilidade clínica ou diagnóstica. A situação atual permanece como Nikolas Rose e Joelle Abi-Rached (2013, p. 138) descreveram, ou seja, "Cada um dos caminhos que a neuropsiquiatria tentou rastrear através do cérebro parece estar correndo, não para as terras altas brilhantes da clareza, mas para a floresta escura, úmida, enevoada e misteriosa da incerteza”.

Então, se a procura por marcadores biológicos tem se tornado infrutífera até o presente momento, indagamo-nos sobre o que teria transformado num estrondoso sucesso mundial uma vertente psiquiátrica denominada de biológica, que não consegue demonstrar a origem biológica dos diagnósticos que constrói desde os anos de 1980.

Partindo desta questão central, consideramos que o imenso sucesso desta Psiquiatria Biológica ao longo do mundo ocidental se deve a uma série de variáveis muito complexas, entre as quais podemos destacar: (a) o surgimento dos primeiros medicamentos psicotrópicos e posterior aumento das suas opçôes disponíveis; (b) as disputas entre as diversas vertentes que compóem o campo psiquiátrico norte-americano; (c) o surgimento e crescimento das indústrias farmacêuticas, que, ao final do século $\mathrm{XX}$, se transformarão em verdadeiros complexos médicos-industriais; (d) o papel desempenhado pelas 
agências governamentais reguladoras dos medicamentos, que passarão a exigir diagnósticos passíveis de serem testados empiricamente para fins de validação e consequente inserção no mercado; (e) bem como a transformação do papel do psiquiatra em meio a esta rede de novas exigências.

Neste artigo, pretendemos analisar a ascensão desta Psiquiatria Biológica a partir das transformaçóes ocorridas no campo psiquiátrico norte-americano com a difusão dos primeiros medicamentos psicotrópicos, bem como pelo surgimento e crescimento das indústrias farmacêuticas.

Este enfoque se justifica na medida em que esta vertente psiquiátrica foi a primeira vertente a se apropriar do uso dos medicamentos psicotrópicos, criando toda uma nomenclatura diagnóstica e uma metodologia de trabalho em consonância com esta ferramenta terapêutica. Criados em 1952, os medicamentos psicotrópicos eram empregados na prática clínica sem qualquer teorizaçáo acerca de seu uso por parte das vertentes psiquiátricas atuantes no campo (SWAIN, 1994). A nosso ver, a Psiquiatria Biológica náo propóe apenas o uso do medicamento psicotrópico na prática clínica, mas ela insere a psiquiatria num modelo de ciência denominado por Bruno Latour (1994, 1997 [com WOOLGAR, S.], 2000) de tecnocientífico, no qual as demais disciplinas biomédicas já estavam se inserindo desde o final da Segunda Guerra Mundial.

Dentro deste novo modelo de ciência, as indústrias, as universidades, as agências governamentais, as agências de publicidade, os médicos, os pacientes e uma série de outros atores passaram a estar interligados numa complexa rede que transcende em muito o cotidiano da psiquiatria de outrora.

Para realização de nossa análise, apoiaremo-nos, especialmente, nos trabalhos de David Healy (2002, 2003) e Edward Shorter (1997), por considerarmos que esses autores traçam um panorama bastante rigoroso acerca da ascensão desta vertente psiquiátrica no cenário norte-americano, o que nos permite realizar nosso debate a partir de dados previamente coletados.

Para iniciar nossa discussão, analisaremos o conceito de tecnociência trabalhado por Latour (1994, 1997 [com WOOLGAR, S.], 2000) e Steven Shapin (2008), para, posteriormente, discutirmos as modificaçôes ocorridas na psiquiatria a partir dos anos de 1980, que inserem esta disciplina dentro de um modelo típico da tecnociência tal qual os autores descrevem. 


\section{Tecnociência: a ciência transformada em profissão}

Latour (2000) considera que os desenvolvimentos tecnológicos adquiridos em meados do século XX deram origem ao que o autor chama de tecnociência. A tecnociência consiste numa ciência baseada na técnica e na tecnologia. Nela, o que está em jogo são os artefatos de inscriçâo, a conexão entre as ideias e as tecnologias e a capacidade política do cientista ou do laboratório de atrair financiamentos e de fazer sua ciência circular através de uma extensa rede, transformando-a em uma caixa preta, que seja capaz de transcender a fronteira do laboratório e de seu próprio grupo de pesquisa, reproduzindo-se como um autômato.

Para Latour (1994, 1997 [com WOOLGAR, S.], 2000), a ligação entre academia-indústria-governo-laboratórios é a característica primordial da tecnociência. Latour aponta ainda que a "pureza científica" fundada tanto na ideia de gênio - que considera a existência de alguma atividade cognitiva especial no cientista - como na ideia de método científico - de que a ciência possuiria um método particular de funcionamento que a faria avançar em direção ao progresso independentemente do contexto social no qual está inserida - ignora toda a rede que sustenta uma descoberta científica.

Segundo Latour (2000), um dos fatores que permite à tecnociência circular numa extensa rede mundial é a mudança de escala produzida através dos artefatos de inscrição, que seriam todo tipo de registro de dados, como tabelas, gráficos, planilhas e estatísticas, por exemplo. Estes artefatos de inscrição tornam os dados produzidos num determinado laboratório (a) móveis para que circulem por outros lugares; (b) os mantêm estáveis para que sejam trazidos e levados sem distorçóes, decomposição ou deterioração; e (c) permitem que sejam combináveis de tal modo que, independentemente da matéria de que sejam feitos, possam ser acumulados, agregados ou "embaralhados como um maço de cartas" (LATOUR, 2000, p. 362).

Isto significa que os artefatos de inscrição permitem que os dados analisados num contexto específico possuam mobilidade, estabilidade e combinabilidade em diversos outros contextos de análise, sejam esses contextos outros laboratórios, outros países, governos etc. É por meio dos artefatos de inscrição que as redes heterogêneas da tecnociência podem se estender dos "centros" à "periferia" e da "periferia" aos "centros". Dentro dos "centros", os dados e números podem ser recombinados de diversas formas, ganhando novos sentidos. 
Dessa maneira, quando um determinado ramo da tecnociência se afirma como profissão e consegue capturar o interesse de outras instituições e do público, ele passa a existir e se expandir de tal modo que em determinado momento nada precisará ser feito para sua expansão devido ao grande número de instituiçôes e atores envolvidos com ele.

Em certo sentido, podemos considerar que o discurso apresentado por Latour (1994, 1997 [com WOOLGAR, S.], 2000) encontra-se bastante alinhado ao de Steven Shapin (2008) no que se refere à transformação da ciência em profissão a partir da tecnociência, bem como ao fato de esta ser uma atividade primordialmente coletiva.

Segundo Shapin (2008), ao longo do século XX ocorre uma profunda mudança no modo de a ciência ser exercida, consequentemente, no modo de o cientista se colocar diante de sua ciência. Tais mudanças teriam sido decorrentes, principalmente, do intercâmbio de informaçóes acadêmicas ocorrido durante a Segunda Guerra Mundial e institucionalizadas durante a Guerra Fria.

A partir deste momento, a pesquisa científica passa a ser uma questão de Estado e o governo americano passa a investir maciçamente neste setor. Simultaneamente, a indústria e as grandes corporaçôes se tornam uma possibilidade de trabalho a mais para o cientista, que, até então, tinha seu ambiente de trabalho restrito aos centros de pesquisa universitários. Nestes novos espaços, o modelo de pesquisa no qual se investe é a pesquisa organizada e aplicada, na qual o trabalho de equipe é dominante. Mas isso não ocorre sem polêmica e muitos cientistas e estudiosos consideram a pesquisa científica organizada e aplicada uma ciência menor (SHAPIN, 2008).

O autor argumenta que é principalmente a partir dos anos de 1970 que todo este movimento iniciado no pós-Segunda Guerra desemboca numa "nova moral da ciência”, na qual esta passa a ser vista como profissão. Assim, a pesquisa científica, que outrora era abordada pelo âmbito da vocação, associada ao desprendimento financeiro e ao trabalho individual, apoiado na ideia de gênio ou do método científico, passa a ser tratada como profissáo, o que inclui questôes salariais e de carreira, bem como trabalho em equipe.

Contudo, vale aqui trazer Latour (2000) para a nossa discussão, pois o autor chama a atenção para o fato de que a tecnociência não pode se expandir da forma aqui relatada em qualquer contexto, devido aos seus altos custos - 
principalmente financeiros, mas não apenas - para construção e consolidação da rede necessária para sua propagação. Assim, países desenvolvidos como os Estados Unidos, que possuem um forte investimento em Pesquisa e Desenvolvimento $(\mathrm{P} \& D)$, têm mais "potencial humano", dinheiro e revistas especializadas que países de pequeno investimento em $\mathrm{P} \& \mathrm{D}$. Por isso, Latour afirma que metade da tecnociência mundial é "negócio americano"; os outros países desenvolvidos trabalham com fatias menores da tecnociência.

Desse modo, existiria uma desigualdade nas condiçôes de realização da tecnociência e sua disseminação que faria com que alguns poucos países produzissem tecnociência propriamente, enquanto outros apenas assimilariam esta tecnociência.

Todo este cenário apresentado por Latour e Shapin, no que se refere às tecnociências, pode ser transposto, em certa medida, para a discussáo que aqui empreendemos acerca da Psiquiatria Biológica e sua indissociável ligaçáo com os medicamentos psicotrópicos, a indústria farmacêutica, órgãos governamentais e agências de fomento. Percebemos ainda a crescente profissionalizaçáo da pesquisa psicofarmacológica e da pesquisa psiquiátrica como parte desta rede. E vale lembrar que essa vertente psiquiátrica nasce nos Estados Unidos, um país com forte investimento em P\&D, e a partir dele se expande rapidamente por diversos países ao longo do mundo, alguns deles como o Brasil, que não possui os recursos financeiros necessários para investir em P\&D como os Estados Unidos.

\section{O surgimento da psicofarmacologia: um novo nicho terapêutico}

O primeiro medicamento psicotrópico, a clorpromazina, foi sintetizado e empregado pela primeira vez no ano de 1952 em pacientes psiquiátricos graves internados no hospital Saint-Anne, que era naquele momento o maior hospital psiquiátrico de Paris. Segundo Healy (2002, 2003), o grande diferencial desta medicação é que ela fazia com que os sintomas delirantes recuassem e, consequentemente, os pacientes se acalmassem, sem, no entanto, ficarem sedados.

Por meio da introdução da clorpromazina no meio psiquiátrico se constituiu o campo da psicofarmacologia no mundo ocidental, que se expande simultaneamente no âmbito da clínica, da pesquisa e do mercado. Esta expansão simultânea em diversos âmbitos e diferentes países ao longo do mundo pode 
ser atribuída ao grande investimento feito pela indústria farmacêutica para a consolidação da psicofarmacologia como um novo campo terapêutico.

Segundo Healy $(2002,2003)$, a clorpromazina teve dificuldades em se inserir no mercado norte-americano, onde outra droga, denominada reserpina, com efeitos semelhantes aos da clorpromazina, já era utilizada. Além disso, nos Estados Unidos, a prática de consultório, dominada pela abordagem psicanalítica, era mais forte que a prática hospitalar, para a qual a clorpromazina estava dirigida.

Contudo, em 1953, a clorpromazina acaba sendo licenciada pela RhonePoulenc para a Smith Kline \& French (SK\& F) como um anti-emético - uma droga para tratar náuseas e vômitos, pois também atuava como um anti-emético.

Assim, a clorpromazina foi lançada como Thorazine, em 1955, nos Estados Unidos. Segundo Healy (2002), ainda que sua prescrição fosse feita não como um psicotrópico, mas sim como um anti-emético, ela foi usada para fins psiquiátricos desde seu lançamento. No segundo semestre de 1955, surge um novo medicamento no mercado norte-americano, o Miltown (meprobamato). Segundo Shorter (1997), esta era uma droga voltada para os casos menos graves, frequentes na prática de consultório, o que fez com que ela tivesse grande aceitação no mercado norte-americano.

A Thorazine e o Miltown foram inicialmente enquadrados na categoria dos tranquilizantes. Devido aos diferentes perfis de atuação destas drogas, a clorpromazina e a reserpina foram consideradas tranquilizantes maiores, enquanto o Miltown e, mais tarde, o Librium, tranquilizantes menores ou leves. Enquanto isso, na Europa, a clorpromazina viria a ser denominada de neuroléptico. Todavia, a partir da década de 1990, os profissionais do mundo todo passariam a denominar, tanto os tranquilizantes maiores quanto os neurolépticos, como antipsicóticos (SHORTER, 1997; HEALY, 2002; HEALY, 2003; RUSSO; VENANCIO, 2006).

A imipramina, que é um tricíclico, foi lançada na Suíça em 1957, com o nome de Tofranil, e, em 1958, esta substância se expande para diversos outros países ao redor do mundo, entre eles os Estados Unidos. Sua principal indicação era para casos de depressão. $\mathrm{Na}$ sequência, vem o Iproniazid (pertencente à classe dos IMAOs - Inibidor de Monoamino-Oxidase), também considerado antidepressivo (HEALY, 2003; RUSSO; VENANCIO, 2006). 
Em 1960, foi lançado o primeiro benzodiazepínico, comercializado como Librium, considerado um tranquilizante e um ansiolítico; seguido por outro benzodiazepínico, o Diazepan, lançado em 1963 e comercializado como Valium. Ambos eram produzidos pela empresa suíça La Roche. Em 1969, o Valium ultrapassa o Librium como a droga mais vendida nos EUA. Shorter (1997) destaca a autonomia e independência da indústria farmacêutica na descoberta e disseminaçáo do Valium:

Como Irving Cohen, um dos psiquiatras que primeiro testou a droga irmã do Valium, o Librium, mais tarde refletiu: "A história do benzodiazepínico (Valium etc.) é essencialmente um modelo de como o agente terapêutico é concebido e trazido por um empreendimento de um fabricante farmacêutico que simplesmente busca encontrar uma droga superior a outras que já estão no mercado." Assim a Roche estava apenas esperando que seus químicos orgânicos a mantivessem a par do jogo (SHORTER, 1997, p. 316-317).

Em 1987, foi lançado o primeiro Inibidor de Recaptação de Serotonina (ISRS), a fluoxetina, conhecida como Prozac, que além de ter sido um grande sucesso de vendas, popularizou o conhecimento acerca dos psicofármacos entre o público leigo e ampliou as possibilidades de tratamento psicofarmacológico em psiquiatria (HEALY, 2002, p. 308; 2003, p. 168).

\section{DSM-III: uma psiquiatria medicamentosa}

Em 1954, a Academia Nacional de Ciências dos Estados Unidos realizou uma conferência que visava estabelecer os métodos adequados de avaliação para as novas farmacoterapias. Esta conferência deu origem ao Serviço Central de Psicofarmacologia do Instituto Nacional de Saúde Mental (NIHM). Esse setor recebeu dois milhôes de dólares do Congresso Americano para avaliar os novos tratamentos e financiar a investigação sobre seus mecanismos de ação. Ele também criou um estudo sobre a clorpromazina em nove hospitais e consolidou a psicofarmacoterapia nos Estados Unidos (HEALY, 2002, p. 280-286).

Em 1962, a Food and Drug Administration (FDA) propôs uma série de emendas que impuseram o método dos Ensaios Clínicos Randomizados (ECRs) como forma de proteger a sociedade contra possíveis erros cometidos nos estudos realizados pela indústria farmacêutica. Estas emendas impostas pela FDA foram uma resposta à chamada "crise da talidomida", ocorrida em 1957 (HEALY, 2002, p. 285-286; HEALY, 2003, p. 24-28; AMARAL, 2004, p. 57-58). 
A realização dos Ensaios Clínicos pode se dar de duas formas: a partir de grandes estudos multicêntricos - o que significa que estes estudos são realizados simultaneamente em diferentes localidades -, ou a partir de estudos de centro único - o que significa que estes estudos são realizados apenas em um local.

Paralelamente a esses debates entre as companhias farmacêuticas, as agências governamentais e o campo médico acerca da eficácia, validade e segurança dos medicamentos psicotrópicos, diversas discussóes tomavam conta da Associação Psiquiátrica Americana (APA) sobre a relevância dos diagnósticos e tratamentos psicanalíticos, hegemônicos na área psiquiátrica daquele país desde o pósSegunda Guerra.

Esses debates culminaram com a queda da psicanálise e a ascensão da Psiquiatria Biológica, que tem como marco de sua hegemonia a publicação do DSMIII (Diagnostic and Statistical Manual of Mental Disorders), em 1980. Podemos considerar que dentro da APA, a queda da psicanálise foi orquestrada pelo grupo de psiquiatras biológicos ligados à Universidade de Washington, em St. Louis. Desde 1970, este grupo propõe a retomada das ideias de Emil Kraepelin (18561926), para quem a construção de uma classificação diagnóstica fidedigna com a observação empírica dos fenômenos clínicos era função central da psiquiatria e pressuposto para a descoberta da etiologia biológica das doenças psiquiátricas (KIRK; KUTCHINS, 1992; YOUNG, 1995; SHORTER, 1997; KUTCHINS; KIRK, 2003; RUSSO; VENANCIO, 2006; CAPONI, 2012; AGUIAR, 2015).

Para Kraepelin, as doenças mentais deveriam ser compreendidas através da analogia com doenças físicas e seguindo um padrão de investigação da medicina mais ampla. Robert Spitzer, organizador do DSM-III, atribui a esse grupo da Universidade de Washington suas "raízes intelectuais" e escolhe toda a comissáo de elaboração desta versão do manual a partir da afinidade com as ideias desse grupo (YOUNG, 1995, p. 99; CAPONI, 2012, p. 167).

Desse modo, consideramos que, assim como Kraepelin, os psiquiatras biológicos percebiam a construção de uma classificação diagnóstica empiricamente embasada como sendo o primeiro passo em direção à descoberta da etiologia das doenças mentais, neste caso, mais precisamente, dos distúrbios mentais.

O DSM-III surge, então, com a proposta de ser a-teórico quanto à etiologia dos distúrbios mentais e de se basear em critérios objetivos de análise dos sinais e sintomas desses distúrbios a fim de construir uma classificação diagnóstica 
descritiva que possuísse confiabilidade e validade junto aos psiquiatras de diferentes países ao longo do mundo, deixando a investigaçáo da etiologia dos distúrbios mentais para pesquisas futuras (ASSOCIAÇÃO AMERICANA DE PSIQUIATRIA, 1986, p. 22).

Essa mudança de paradigma na psiquiatria representada pela publicação do DSM-III, e consolidada com a publicação do DSM-IIIR (1987) e DSM-IV (1994), é fruto de uma série de fatores que vão desde disputas internas ao campo psiquiátrico até a pressão de agências governamentais, das seguradoras de saúde, das famílias de pacientes e da indústria farmacêutica sobre os meios psiquiátricos (SHORTER, 1997; YOUNG, 1995; SERPA, JR. 1998; HEALY, 2002, 2003; RUSSO \& VENANCIO, 2006; CAPONI, 2012).

Se nos detivermos, a título metodológico, especialmente na influência da indústria farmacêutica sobre os meios psiquiátricos, perceberemos que o formato diagnóstico proposto pelo DSM-III possui grande relevância para as demandas da indústria farmacêutica por parâmetros classificatórios de diagnósticos que pudessem ser reproduzidos nos Ensaios Clínicos Randomizados (ECRs) exigidos pela FDA.

Isto ocorre porque para que os Ensaios Clínicos Randomizados (ECRs) sejam realizados, é necessário que existam diagnósticos bem delimitados, assim como uma população homogeneamente diagnosticada. E, por sua vez, uma população só poderia ser homogênea em termos de diagnóstico se houvesse um diagnóstico tão específico a ponto de ser replicável nas diferentes populaçôes a serem pesquisadas, o que a psicanálise jamais poderia oferecer e o DSM-III pôde.

Segundo Healy (2002, 2003), como as exigências da FDA para licenciamento de medicamentos costumam servir de parâmetro para as indústrias farmacêuticas de todo o mundo ocidental, devido à importância do mercado norte-americano, esta afinidade entre o formato do manual e as exigências da FDA teria agilizado a expansão da Psiquiatria Biológica ao longo do mundo.

Contudo, a transposição do modelo avaliativo dos Ensaios Clínicos Randomizados (ECRs) para o campo psiquiátrico apresenta uma série de implicaçôes. Como Serpa Jr. (1998) argumenta, mesmo com os inegáveis avanços da genética e dos estudos sobre o cérebro, não é possível precisar em nenhum destes sistemas a origem dos distúrbios mentais, ainda que estes sejam cada vez mais significados a partir de um vocabulário biológico/fisicalista. 
Por não haver marcador biológico específico para os distúrbios/transtornos mentais, os medicamentos psiquiátricos não podem funcionar como a maioria dos medicamentos utilizados para tratar doenças que possuem este tipo de marcador biológico sobre o qual atuar. Assim, os psicotrópicos, diferentemente dos antibióticos, por exemplo, possuem uma atuação mais difusa sobre o organismo do paciente. Segundo Healy:

As descobertas farmacológicas dos anos de 1950 reacenderam os debates sobre os papéis das intervençôes específicas e não específicas para o tratamento de condiçôes de doenças que iam desde doenças infecciosas até a esquizofrenia. Não obstante os efeitos dramáticos da clorpromazina, em alguns casos, a maioria dos médicos inicialmente viam a droga como uma intervenção relativamente inespecífica. As perguntas eram em que medida o trabalho poderia ter a sua eficácia demonstrada de forma convincente quando a cura era raramente alcançada. [...] (HEALY, 2002, p. 279).

Seguindo esta linha de raciocínio, o autor argumenta que os Ensaios Clínicos acabam por fazer com que uma resposta, mesmo que sutil, de um grupo diagnosticado com alguma das categorias de distúrbio/transtorno descrita no DSM-III ou em suas ediçóes seguintes, para uma droga psicotrópica Y signifique aparentemente que a entidade de doença $\mathrm{X}$ estaria respondendo à droga psicotrópica $\mathrm{Y}$ da mesma forma que culturas de bactérias respondem a antibióticos. O que não é verdadeiro. Segundo Healy:

Os ECR's mudaram a maneira como os psiquiatras olham para os pacientes. Estes tipos de ensaios funcionam melhor quando existe uma população de doentes homogênea. Este fato favorece a montagem de entidades de categorias de doença, como a fobia social, o transtorno de ansiedade generalizada, ou o transtorno do pânico. Uma resposta dessas entidades de doença aparentemente discretas para uma droga, então, cria uma ilusão de especificidade [...] (HEALY, 2002, p. 284).

Além disso, como Márcia Angell (2007) e Healy (2002) argumentam, os Ensaios Clínicos são extremamente dispendiosos, o que permite que a indústria farmacêutica seja a principal responsável por sua realização e pela divulgação de seus resultados. Assim, a indústria farmacêutica pode divulgar apenas os resultados positivos e ocultar os resultados negativos (BENTALL, 2009; HEALY, 2002, 2003, 2004; GREENBERG, 2010; KIRSCH, 2009; ANGELL, 2007; RICHARD, 2009; GUPTA, 2014; KIRMAYER; RAIKHEL, 2009; WHITAKER, 2010; DUMIT, 2012; GOLDACRE, 2013).

Vemos ainda que nesse período muitas revistas e jornais da área psiquiátrica passam a se sustentar graças à propaganda de medicamentos feitas em suas 
páginas, e muitos centros de excelência e pesquisadores reconhecidos na área psiquiátrica ou psicofarmacológica passam a ter suas pesquisas financiadas pela indústria, podendo receber uma encomenda de alguma pesquisa ou mesmo receber um determinado modelo de pesquisa pronto, que teriam apenas que aplicar. Os pesquisadores poderiam também ser contratados como consultores pelas empresas de medicamentos ou se tornarem pesquisadores da própria empresa (HEALY, 2002, 2003; ANGELL, 2007).

Esse financiamento da indústria farmacêutica de pesquisas biomédicas gera conflitos de interesses que os médicos e pesquisadores muitas vezes preferem não divulgar. Esta situação tem levado a um enfraquecimento significativo da confiança pública e a intensas discussóes sobre a melhor forma de regulamentar esta área da profissão médica (ANGELL, 2007; GRANDE, 2010; GREEN et al., 2012, WAZANA, 2000). A força motriz da indústria farmacêutica não pode ser meramente uma questáo de economia e medicina, mas deve ser também de ética profissional.

\section{Considerações finais: A rede médico-industrial norte- americana}

Se retomarmos nosso objeto de estudo, que é a Psiquiatria Biológica e sua relação com a psicofarmacologia, veremos que é impossível falar de psicofarmacologia sem falar da indústria farmacêutica, visto o monopólio que esta mantém no que se refere à pesquisa, produção e difusão dos medicamentos psicotrópicos desde seu surgimento.

Isto se agrava ainda mais porque quando o DSM-III surge, a Psiquiatria Biológica demonstra considerar este manual classificatório apenas como um passo inicial em direção à descoberta da etiologia dos transtornos mentais, que, segundo o próprio manual, deveria ser investigado em pesquisas futuras. Contudo, o segundo passo não foi dado até a atualidade por limites em relação a estas descobertas. Assim, o DSM-III e seus sucessores (já na quinta edição), bem como os psicofármacos, acabaram se tornando a principal fonte de legitimação da Psiquiatria Biológica e seu maior expoente.

A partir deste dado, percebemos que a uniáo entre estes dois nichos terapêuticos - Psiquiatria Biológica e Psicofarmacologia - gerou um ciclo de "retroalimentação" que dificulta saber onde uma começa e onde a outra termina: 
a indústria de medicamentos psicotrópicos precisa dos diagnósticos bem

demarcados que a Psiquiatria Biológica oferece para realizar suas pesquisas e para a aprovação dos seus medicamentos junto à FDA, bem como precisa da prescrição dos medicamentos por parte dos psiquiatras para que continue se expandindo.

Por outro lado, a Psiquiatria Biológica utiliza os medicamentos psicotrópicos como opção de primeira escolha em sua terapêutica e se apoia nos financiamentos da indústria farmacêutica para realizar suas pesquisas e divulgar seus diagnósticos, bem como sua visão de ciência de modo mais amplo. E quanto mais a visão de ciência, diagnósticos e psiquiatras-pesquisadores da Psiquiatria Biológica estiverem consolidados, mais confiabilidade e validade ganha o uso do medicamento psicotrópico, que por ser a principal via de tratamento desta vertente gera, por sua vez, mais lucro para as indústrias farmacêuticas, que podem financiar cada vez mais centros de excelência, pesquisas, psiquiatraspesquisadores, e assim por diante.

Todo este circuito deixa as fronteiras entre a psicofarmacologia, a indústria farmacêutica e a Psiquiatria Biológica cada vez mais diluídas e, até o presente momento, todas estas áreas fortalecidas. E, curiosamente, as pesquisas acerca da etiologia biológica dos distúrbios/transtornos mentais desta vertente psiquiátrica autodenominada biológica percorrem o caminho inverso desta expansão, ficando cada vez mais enfraquecidas e nebulosas em meio a esta rede.

Ao mesmo tempo que é impossível ignorar o peso da indústria farmacêutica sobre a área de pesquisa com medicamentos psicotrópicos, também não podemos ignorar que as pesquisas na área da saúde em geral, e em psiquiatria em particular, não poderiam ficar restritas aos meios acadêmicos-universitários diante da importância que esta área ocupa em nossa sociedade.

No caso específico da psiquiatria, consideramos que ainda que os métodos avaliativos acerca do emprego dos psicofármacos na clínica psiquiátrica apresentem muitas limitaçôes e estejam excessivamente dependentes do financiamento e controle da indústria farmacêutica, bem como restrita aos países com forte investimento e $\mathrm{P} \& \mathrm{D}$, devido aos seus altos custos, isto não significa que outros métodos avaliativos, modelos de financiamento e recursos regulatórios mais eficazes sobre as pesquisas com psicofármacos não possam ser produzidos nem que todo conhecimento produzido acerca desta classe de medicamentos encontra-se invalidado. 
Além disso, os psicofármacos são empregados por psiquiatras do mundo todo, independentemente de sua filiação teórica, demonstrando eficácia no cotidiano da prática clínica. Contudo, até o presente momento não costumam ser estudados, e muitas vezes sequer debatidos, por psiquiatras que não sejam representantes da Psiquiatria Biológica.

Desse modo, devemos refletir também sobre o fato de que as outras vertentes do campo psiquiátrico se recusam ou, no mínimo, se omitem no que se refere à realização ou proposição de pesquisas com esta classe de medicamentos. Assim, vemos se reproduzir em outros termos a dicotomia entre discurso e prática denunciada por Gladys Swain (1994) no cenário psiquiátrico dos anos de 1960, quando psiquiatras psicanalistas ou psiquiatras ligados a uma vertente da psiquiatria mais social, por exemplo, utilizavam os psicotrópicos no cotidiano da clínica, mas só debatiam publicamente questóes de ordem psicológica e social.

Esta situação só faz ampliar o monopólio da indústria farmacêutica e da Psiquiatria Biológica na produção de conhecimento acerca dessa classe de medicamentos, bem como dificulta que os profissionais de outras vertentes psiquiátricas tenham conhecimento suficiente sobre este tema para ampliar o debate acerca das questóes éticas implicadas na produção de pesquisas com psicofármacos, assim como sobre seu valor de uso na clínica psiquiátrica.

\section{Referências}

AGUIAR, M. Programa de Ansiedade e Depressão: o desenvolvimento da Psiquiatria Biológica no Instituto de Psiquiatria da Universidade Federal do Rio de Janeiro (1984-1998). $275 f$. Tese (Doutorado em História das Ciências e da Saúde) - Casa de Oswaldo Cruz, Fundação Oswaldo Cruz, Rio de Janeiro, 2014.

AGUIAR, M.P. Ascensão e consolidação da psiquiatria biológica norte-americana: uma análise histórica. Mnemosine. Rio de Janeiro: UERJ, v. 11, no 1, p. 227-257, 2015.

AMARAL, A. A psiquiatria no divã: Entre as ciências da vida e a medicalização da existência. Rio de Janeiro: Relume Dumará, 2004. 166 p.

ANGELL, M. A verdade sobre os laboratórios farmacêuticos. Rio de Janeiro: Record, 2007. $186 \mathrm{p}$.

ASSOCIAÇÃO AMERICANA DE PSIQUIATRIA. Manual Diagnóstico e Estatístico de Doenças Mentais DSM-III. Lisboa: Editora Portuguesa de Livros Técnicos, 1986. 473 p. . Manual Diagnóstico e Estatístico de Doenças Mentais DSM-III-R. São Paulo: Manole, 1989. $602 \mathrm{p}$. 

ARTMED, 2000. 880 p.

BENTALL, R. Doctoring the Mind: Why Psychiatric Treatments Fail. New York: New York University Press, 2009. 304 p.

BEZERRA JR., B. Naturalismo como anti-reducionismo: notas sobre o cérebro, mente e subjetividade Cadernos IPUB. Rio de Janeiro, v. VI, n. 18, p. 158-177, 2000.

BYNUM, W.F.; PORTER, R.; SHEPHERD, M. (Org.). The Anatomy of Madness: Essays in the History of Psychiatry, Vol. I: People and Ideas, Vol. II: Institutions and Society. London: Routledge, 1985. 264 p. e 283 p.

CAPONI, S. Loucos e Degenerados: uma genealogia da psiquiatria ampliada. Rio de Janeiro: Editora FIOCRUZ, 2012. 210 p.

CASTEL, R. A Ordem Psiquiátrica: a idade de ouro do alienismo. Rio de Janeiro: Editora Graal, 1978. 329 p.

CHARNEY, D. et al. Neuroscience research agenda to guide development of a pathophysiologically based classification system. In: KUPFER, D.J.; FIRST, M.B.; REGIER, D.A. (Org.). A Research Agenda for DSM-V, 31-84. Washington, DC: American Psychiatric Association, 2002.

DINIZ, D. et al. (Org.). Ética em pesquisa: temas globais. Brasília: LetrasLivres: Editora $\mathrm{UnB}, 2008.404 \mathrm{p}$.

DUMIT, J. Drugs for Life: How Pharmaceutical Companies Define our Health. Durham: Duke University Press, 2012. 280 p.

FOUCAUlT, M. A grande internação. In: História da Loucura. São Paulo: Editora Perspectiva, p. 45-79, 2002.

GABRIELI, J.; GHOSH, S.; WHITFIELD-GABRIELI, S. Prediction as a Humanitarian and Pragmatic Contribution from Human Cognitive Neuroscience. Neuron, v. 85, n. 1, p. 11-26, 2015.

GOLDACRE, B. Bad Pharma: How Drug Companies Mislead Doctors and Harm Patients. New York: Faber \& Faber, 2013. 448 p.

GRANDE, D. Limiting the Influence of Pharmaceutical Industry Gifts on Physicians: Self-Regulation or Government Intervention?" Journal of General and Internal Medicine, v. 25, n. 1, 2010, p. 79-83.

GREEN, M.J. et al. Do gifts from the pharmaceutical industry affect trust in physicians? Family Medicine, n. 44, p. 325-31, 2012.

GREENBERG, G. Manufacturing Depression: The Secret History of a Modern Disease. New York: Simon \& Shuster, 2010. 448 p. 
GUPTA, M. Is Evidence-based Psychiatry Ethical? Oxford: Oxford University Press, 2014. $211 \mathrm{p}$.

HEALY, D. The Creation of Psychopharmacology. Cambridge, Mass. and London: Harvard University Press, 2002. 469 p.

. The anti-depressant era. Cambridge: Harvard University Press, 2003. 317 p.

HEALY, David. Let Them Eat Prozac: The Unhealthy Relationship between the Pharmaceutical Industry and Depression. New York: New York University Press, 2004. 351 p.

HYMAN, S.E. A glimmer of light for neuropsychiatric disorders. Nature, n. 455, p. 890893, 2008.

INSEL, T. et al. Research domain criteria (RDoC): toward a new classification framework for research on mental disorders. American Journal of Psychiatry, n. 167, 2010, p. 748-751.

INSEL, T.R. Transforming diagnosis., 2003. Disponível em: <www.nimh.nih.gov/about/ director/2013/transforming-diagnosis.shtml>.

KAPUR, S.; PHILLIPS, A.G.; INSEL, T. Why has it taken so long for biological psychiatry to develop clinical tests and what to do about it? Molecular Psychiatry, v. 17, n. 12, p. 11741179, 2012.

KIRK, S.A.; KUTCHINS, H. The Selling of DSM: The Rhetoric of Science in Psychiatry. New York: Aldine, 1992. 270 p.

KIRMAYER, L.J.; RAIKHEL, E. Editorial: from Amrita to substance D: psychopharmacology, political economy, and technologies of the Self. Transcultural Psychiatry, n. 46, p. 5-15, 2009.

KIRMAYER, L.J.; CRAFA, D. What kind of science for psychiatry. Frontiers in Human Neuroscience, n. 8, art. 435, p. 1-12, 2014.

KIRSCH, I. The Emperor's New Drugs: Exploding the Antidepressant Myth. London: Bodley Head, 2009. 240 p.

KUTCHINS, H.; KIRK, S.A. Making Us Crazy: DSM: The Psychiatric Bible and the Creation of Mental Disorders. New York: Free Press, 2003. 304 p.

LATOUR, B. Ciência em ação: como seguir cientistas e engenheiros sociedade afora. São Paulo: Editora UNESP, 2000. 438 p.

. Give me a laboratory and I will raise the world. In: KNORR-CETTINA, K.; MULKAY, M. (Editors). Science observed. perspectives on the social study of science. Beverly Hills: Sage, 1983, p. 141-170.

- Jamais Fomos Modernos: ensaio de antropologia simétrica. Rio de Janeiro: Editora 34, 1994. $150 \mathrm{p}$.

LATOUR, B.; WOOLGAR, S. Vida de Laboratório: a produçâo dos fatos científicos. Rio de Janeiro: Relume Dumará, 1997. 310 p. 
MAYBERG, H.S. Neuroimaging and Psychiatry: The Long Road from Bench to Bedside. Hastings Center Report, 44, p. S31-S36, 2014.

PIGNARRE, P. O que é medicamento? Um objeto estranho entre ciência, mercado e sociedade. Rio de Janeiro: Editora 34, 1999. 152 p.

RELMAN, A. The New Medical Industrial-Complex. New England Journal of Medicine, n. 303, p. 963-970, 1980.

ROSE, N. What is diagnosis for? Paper for a Conference on DSM-5 and the Future of Diagnosis. 2013b. Disponível em: <http://nikolasrose.com/index.php/lectures/>.

ROSE, N.; ABI-RACHED, J.M. Neuro: The New Brain Sciences and the Management of the Mind. Princeton: Princeton University Press, 2013. 352 p.

ROSENBERG. C.E. The tyranny of diagnosis: specific entities and individual experience. The Milbank Quarterly. Oxford, UK., v. 80, n. 2, p. 237-260, 2002.

. Contested Boundaries: psychiatry, disease, and diagnosis, Perspectives in Biology and Medicine, v. 49, n.3, p. 407-424, 2006.

RUSSO, J.; VENANCIO, A.T. Classificando pessoas e suas perturbaçóes: a "revolução terminológica” do DSM III. Revista Latinoamericana de Psicopatologia Fundamental, v. IX, n.3, p. 460-485, 2006.

SCULL, A (Org.). Madhouses, Mad-Doctors, and Madmen: The Social History of Psychiatry in the Victorian Era. Philadelphia: University of Pensilvania Press, 1981. 384 p.

. The most solitary of afflictions: madness and society in Britain, 1700-1900. London and New Haven: Yale University Press, 2005. 448 p.

. Madness in Civilization: A Cultural History of Insanity, from the Bible to Freud, from the Madhouse to Modern Medicine. Princeton: Princeton University Press, 2015. 448 p.

SERPA JR., O.D. Mal-estar na natureza: estudo crítico sobre o reducionismo biológico em psiquiatria. Rio de Janeiro: Te Corá, 1998. 372 p.

. Mente, cérebro e perturbação mental. Cadernos do IPUB. Rio de Janeiro: UFRJ, v. VI, n. 18, p. 144-157, 2000.

SHAPIN, S. Discipline and bounding: the history and sociology of science as seen through the externalism-internalism debate. History of Science, v. 4, n. 90, p. 333-369, 1992.

. The scientific life: A moral history of late modern vocation. Chicago: The University of Chicago Press, 2008. 486 p.

SHORTER, E. A History of Psychiatry: The Era of asylum to the age of Prozac. New York, USA: John Wiley \& sons, 1997. 436 p.

SWAIN, G. Chimie, cerveau, esprit et societé: paradoxes epistemologiques des psychotropes en medicine mentale. In: SWAIN, G. Dialogue avec l'insensé. Paris: Ed. Gallimard, 1994, p. 261-281. 
VALENSTEIN, E.S. Blaming the brain: The truth about drugs and mental health. New York: Free Press, 1998. 304 p.

VIANNA, C.M.M. Estruturas do Sistema de Saúde: do Complexo Médico-Industrial ao Médico Financeiro. Physis: Rev. Saúde Coletiva, Rio de Janeiro, v. 12, n. 2, 2002, p. 375-390. YOUNG, A. The Harmony of Illusions: Inventing Post-Traumatic Stress Disorder. New Jersey: Princenton University Press, 1995. 327 p.

WALSH, P. et al. Search of biomarkers for autism: scientific, social and ethical challenges. Nature Reviews Neuroscience, n. 12, p. 603-612, 2011.

WAZANA, A. Physicians and the pharmaceutical industry: is a gift ever just a gift? Journal of the American Medical Association, v. 283, n. 3, 2000, p. 373-380.

WHITAKER, R. Anatomy of an Epidemic: Magic Bullets, Psychiatric Drugs, and the Astonishing Rise of Mental Illness in America. New York: Crown, 2010. 432 p.

\section{Notas}

${ }^{1} \mathrm{O}$ surgimento da psiquiatria recebe diferentes interpretaçôes, mas é um consenso entre os estudiosos da área que é a partir do século XVIII que esta se constitui como uma disciplina pertencente ao campo médico (CASTEL, 1978; FOUCAULT, 2002; SERPA Jr., 1996; SCULL, 1981, 2005; SHORTER, 1997).

${ }^{2} \mathrm{O}$ modo como são nomeados àqueles que recebem tratamento psiquiátrico varia de acordo com a corrente teórica a partir da qual estamos partindo. A título de uniformização, utilizaremos neste trabalho a denominação "doença mental", com exceção de quando estivermos nos referindo à nomenclatura do DSM-III e DSM-III-R, que, no Brasil, adotaram o termo "distúrbio mental", e o DSM-IV, publicado posteriormente, em 1994, que passa a chamar estes "distúrbios" de "transtornos mentais". De todo modo, vale esclarecer que na versão norte-americana do DSM-III, DSM-III-R, DSM-IV e DSM-V, o termo se mantém sempre o mesmo: disorder. Para mais informações, ver: Associação Americana de Psiquiatria (1986 e 2002), Shorter (1997), Healy (2002 e 2003), Young (1995) ,Rosenberg (2006) e Russo e Venancio (2006).

${ }^{3}$ Verificar a nota de fim 2.

${ }^{4}$ Referimo-nos a marcador biológico em termos de origem cerebral destes distúrbios. Além disso, como argumenta Allan Young (1995), a maioria das vezes em que um distúrbio/transtorno mental teve um marcador biológico encontrado, este saiu do domínio da psiquiatria e passou a fazer parte do campo da neurologia.

${ }^{5}$ Ainda que esta vertente denominada de Psiquiatria Biológica, da qual trataremos neste artigo, seja apenas o expoente de maior alcance - em termos de expansão das suas ideias e categorias diagnósticas ao longo do mundo ocidental -, ela não deve ser vista como um corpo homogêneo nem a única linha a estudar as doenças mentais pelo viés biológico. Não entraremos neste debate para não nos perdermos de um dos argumentos centrais deste artigo, que é justamente a força que esta vertente psiquiátrica alcançou sob a nomenclatura de Psiquiatria Biológica sem que a etiologia biológica dos distúrbios por ela construídos e estudados tivesse sido demonstrada.

${ }^{6} \mathrm{O}$ conceito de complexo médico-industrial é utilizado por Cid Vianna para se referir às múltiplas e complexas relaçóes estabelecidas entre os atores do setor da saúde com os demais setores da economia. Para mais informações: Vianna (2009) e Relman (1980). 
${ }^{7}$ Contudo, é relevante destacarmos que Edward Shorter apresenta a mudança de referenciais na psiquiatria, referida no texto, com uma abordagem diferente da que David Healy utiliza e com a qual nos alinhamos. Shorter considera que a história da psiquiatria foi dominada por abordagens biológicas e que a influência das teorias morais-humanistas, como a psicanálise, por exemplo, foram exceções na história desta disciplina. Assim, nós nos apoiamos em sua historiografia, mas consideramos que seja constitutivo do campo psiquiátrico esta circularidade entre as teorias hegemônicas na área. Para mais informaçôes, ver: Shorter (1997) e Healy (2002 e 2003).

${ }^{8}$ Advindo do campo da cibernética, o conceito de caixa preta é utilizado para se referir a um conjunto de comandos que de táo complexo deixam de ser explicados e passam a ser tratados como um fato a partir do qual se parte. Bruno Latour se apropria desse conceito para se referir ao momento em que uma dada teoria científica se consolida de tal forma que todo o percurso que levou à sua construção é esquecido, o que a transforma num fato. Cf. Latour (2000, p. 14 e 216).

${ }^{9}$ Para mais informaçôes, recomendamos: Young (1995), Shorter (1997) e Healy (2002 e 2003).

${ }^{10}$ Para uma história das modificaçóes ocorridas na nomenclatura dos medicamentos psicotrópicos, recomendamos especialmente o livro The Antidepressant Era, de David Healy (2003).

${ }^{11}$ A Food and Drug Administration (FDA) é um órgão governamental dos Estados Unidos, responsável pelo controle dos alimentos, suplementos alimentares, medicamentos, cosméticos, equipamentos médicos e materiais biológicos. A FDA foi criada em 1862, contudo só passou a ter a atual designação em 1930.

${ }^{12}$ Esta crise teve início quando o composto denominado de talidomida foi lançado com o nome de Cortegan, na Alemanha, para ser usado como sedativo e tranquilizante. Nos testes pré-clínicos, ela demonstrou ser um composto seguro, mas ao ser utilizado por mulheres grávidas gerou uma série de deformidades congênitas em milhares de fetos - seis mil aproximadamente.

${ }^{13}$ Para mais informaçốes sobre o modo de funcionamento dos Ensaios Clínicos Randomizados (ECRs), indicamos especialmente: Pignarre (1999) e Amaral (2004).

14 Robert Spitzer (1932-2015) foi um psiquiatra de orientaçấo biológica que ocupou o cargo de presidente da Comissão de Nomenclatura e Estatística, chamada também de Força Tarefa (Task Force on Nomenclature and Statistics da American Psychiatry Associassion), criada para elaboração do DSM-III.

${ }^{15}$ Vale destacar, que ainda que a obra de Serpa Jr. date dos anos de 1998, seu argumento permanece atual, como vimos na introduçáo deste artigo. De qualquer modo, vale destacar que náo haver marcador biológico para os distúrbios mentais não significa que alguns "subtipos" de distúrbios não possam ser influenciados por disfunçóes fisiológicas, como é o caso, por exemplo, de um subtipo de depressão ocasionada por hipotireoidismo. Contudo, este tipo de disfunção não explica todos os casos de depressão que continuam sem uma causa fisiológica ou cerebral demarcada e nem todo distúrbio/ transtorno mental possui este tipo de disfunção fisiológica.

${ }^{16}$ Para uma discussão sobre os dilemas éticos na área de pesquisa global, indicamos Diniz et al. (2008).

${ }^{17}$ M.P. Aguiar foi responsável pela construção do corpo do artigo, realizando o debate acerca da relação entre a Psiquiatria Biológica e a Psicofarmacologia como parte de uma rede tecnocientífica. Parte deste debate se apoia nos dados coletados em sua tese de doutorado (AGUIAR, 2014). F.J.G. Ortega colaborou com a ampliação do debate realizado sobre a relação entre a Psiquiatria Biológica e a Psicofarmacologia a partir de discussôes sobre a etiologia biológica dos distúrbios/transtornos psiquiátricos na atualidade, bem como da relação da Psiquiatria Biológica com a indústria farmacêutica. Adicionou bibliografia pertinente ao tema. 


\section{Abstract}

\section{Biological Psychiatry and Psychopharmacology: a techno-scientific network in debate}

Biological Psychiatry emerged in the 1980s in the United States with the publication of DSM-III (Diagnostic and Statistical Manual of Mental Disorders). This manual intends to be a-theoretical and to provide objective descriptions of "mental disorders" by using diagnostic criteria that do not include discussions about the aetiology of these "disorders". Hence and despite the "biological" label conferred to this branch of psychiatry, there is no explicit concern to ascertain the possible biological origins of "mental disorders" in DSM-III. In this paper we intend to examine the modifications in North American Psychiatry following the introduction of the first psychotropic medications and the subsequent role played by the pharmaceutical industry in this process. Thus, what is under analysis in our article is that, by appropriating psychotropic drugs as the first therapeutic option, Biological Psychiatry creates an alliance with the pharmaceutical industry that inserts it into a technoscientific network that makes it difficult to know the limits between Biological Psychiatry, Psychopharmacology and the Pharmaceutical industry.

> Keywords: Biological Psychiatry; Psychopharmacology; network; technoscience. 